\title{
The Design and Realization of the Household Intelligent Alarm System
}

\author{
Guo Hong Tao \\ (North China University of Water Conservancy and Hydroelectric Power, \\ Zhengzhou 450011, China)
}

\begin{abstract}
Security issue of our activity and residential zone is becoming more and more important. Developing a new home alarm system based on the public telephone network is very important to the protection of life and property safety of residents and improving the rapid response capability of departments, such as public security, fire control, medical treatment. This paper proposes a solution to design and realization the system. The whole system based on microcontroller AT89C51 is divided into hardware system and software system. Hardware system consists of pyroelectric infrared sensor, alarm mainframe, dial circuit, voice circuit, telephone interface circuit, key interface circuit. And software system includes master control modules, alarm processing modules, interrupt processing modules, dial modules, voice modules and keyboard input modules. When Infrared sensor detects alarm signal such as fire, housebreaking and emergency pulses of elder, the microcontroller receives interrupt request from prober and controls telephone interface circuit to finish simulating off-hook. Using DTMF (dual-tone multi-frequency) dial circuit to realize auto dial and voice call to play prerecorded voice messages to inform the host of the house to take measures timely. The system without rewiring is particularly applicable in the decoration and inconvenient for the user. This system has been validated by experiments, and will be a fast and effective practical solution for users.
\end{abstract}

Key words: Alarm system, microcontroller, infrared sensor

\section{Introduction}

With the continuous improvement of people's living standard, people pay more attention to home security regularly. It is expected to install systems with the function of security and danger alarm in family house so that the host could master the occurred accident in time [1-3]. Intelligent telephone alarm system with advantages of high reliability, low cost and easy popularization is developed to fit this requirement. The system with AT89C51 as the core, combining with technologies including DTMF(Dual-tone multi-frequency) data transmission technology, voice chip ISD1420, memory chip AT24C01 and pyro electric infrared sensor [4-5] to realize the monitoring of fire and theft, using public communication network as transmission medium to achieve automated dial-up alarm and play voice signals transcribed already.

\section{System Composition and the Basic Principle}

Alarm system is composed by the following elementary function modules: leading end prober, automatic on-hook and off-hook, dual sound signal decode, voice record-replay and master controller. Software and hardware are divided to optimize the combination of structure, performance, volume and cost. Hardware supports the prober, automated off-hook and on-hook, telephone interface circuit, DTMF dial and recording sound; ringing tone and busy tone count, analysis and process of input information are relied on software. Main control part is composed by microcontroller. System structure diagram is 
shown in Figure 2.1.

Prober finishes the monitoring for pyroelectric infrared signal of human body and data acquisition, $\mathrm{A} \backslash \mathrm{D}$ converter, comparative judgment. It will export alarm switching signal to autoalarm mainframe when the actual parameter reaches the setting alarm numeric。

microcontroller (AT89c51 used in this system) as the central device and "heart" of the whole system, receives the alarm signal, controls and coordinates the proper functioning of functional modules.

Importing prestored phone numbers of users by keyboard, storing original parameters and important information of system in EEPROM (Electrically Erasable Programmable Read-Only Memory).

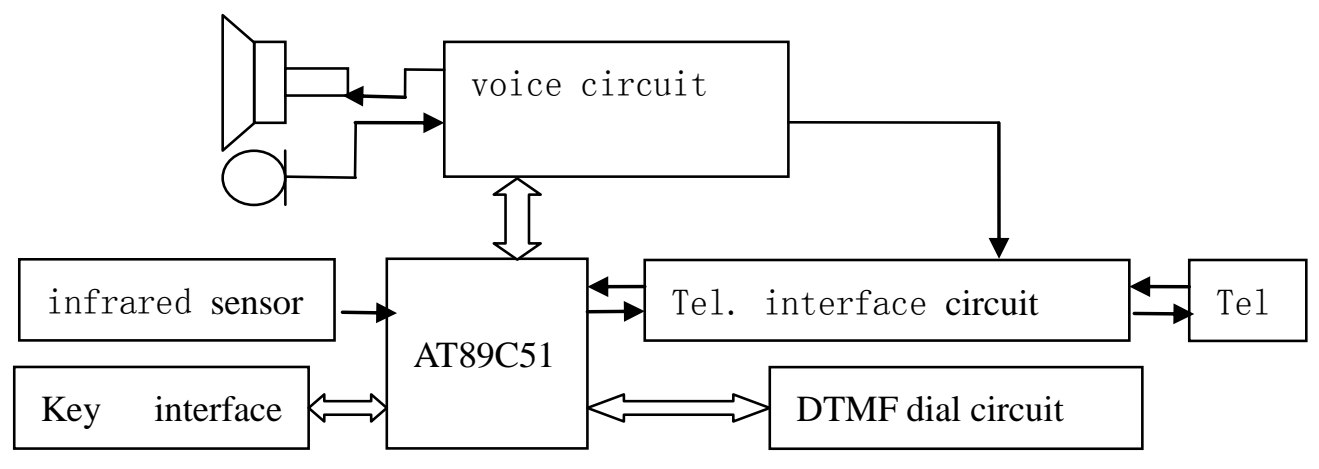

Figure 2.1. System Architecture

Dial circuit uses the coding integrated chip MT8888 of company MITEL to transmit phone numbers, and matches with microcontroller to monitor signal tone of telephone wire. Voice circuit adopts high fidelity RP (record playback) integrative speech chip ISD1420 of company ISD in America to prestore the voice prompt, record and transmit corresponding voice prompt according to the situation.

Alarm prober should be installed in the place that needs precaution. Usually, in dormant state while in protective state when the users start the control monitor and prober when they are out. As long as someone goes by the detection range, alarm prober will send out alarm (suspend) signal at once, autoalarm mainframe notarizes after receiving the events (polling interrupted signals repeatedly) and reads corresponding alarm call number from EEPROM (Electrically Erasable Programmable Read-Only Memory), if no errors, then dials the ordered number (phone number, office number) to make long-distanced dial alarm by dual tone multiple frequency dial-up chip. After the off-hook, autoalarm mainframe enables the voice circuit and passes on the ordered voice messages through telephone wire.

\section{System Hardware Circuit Design}

Pyroelectric infrared prober ,alarm mainframe, dial circuit, voice circuit, phone interface circuit, button interface circuit make up the whole system.

\subsection{Pyro electric Infrared Prober}

Pyroelectric infrared prober transforms infrared ray getting from moving human body into low-frequency electrical signals associated with the velocity of movement, distance and direction. By using pyroelectric infrared sensing, signal processor BIS0001 can test stealing signal. 


\subsection{Alarm Host}

The mainframe of alertor is composed by microcontrollers AT89c51 and 8255A. Interface PO is the data bus, linking with the 8255A and MT8888. DTMF signal and voice signal switch by linkage of P1.6 and dial voice circuit, P1.6 connects with playback PLAYL/ of IAD1420 voice chip, low level valid.P1.7 stimulates off-hook and on-hook control, implement system and work switchover of substation.

P2.0 and RSO (railway sorting office) of MT8888 are joined to choose internal memory.P2.1, P2.2 and A0, A1 address wire, P2.5 and spot alarm bell are together, P2.6 and P2.7 are separately linked with CS gate of 8255A and MT8888.TI port links with IRQ/CP of MT8888 to count the number of monitoring phone voice square signals, INTO port and infrared prober output end connect in negater to receive alarm signal and interrupt. WR, RD and that of MT8888, 8255A are together to command the read of MT8888, 8255A.

\subsection{Dial Circuit}

MT8888, a strong DTMF dispatch and reception device with Intel microprocessor port, is useful for paging, interchanger and debit card system, mobile and digital communication, interconnected dialer and computer. Main functions: (1) intact DTMF transmit and reception;(2) super-speed Intel microprocessor port;(3) work for automatic frequency burst mode; (4)adjust guard time; (5) calling tone detected-30dBm.

The interface circuit of MT8888 and microprocessor AT89c51 of the system are as shown in figure3.1.Its receiving unit adopts single-ended input, consisting of R1, R2 and $\mathrm{C} 1$, input voltage gain is $\mathrm{R} 2 / \mathrm{R} 1=1$, by changing $\mathrm{R} 2$ can adjust gain of input signal; $\mathrm{C} 5$, $\mathrm{R} 3, \mathrm{C} 2$ and $\mathrm{X} 1$ make up the sending unit; R4 and $\mathrm{C} 3$ form the control section. DTMF IN, DTMF OUT link with the phone interface circuit. Pins like /WR, /RD separately connect with that of microcontroller to control read-write of the chip. ROSR (read-only-storage) pin and P2.0 are together to finish the chosen internal memory.

\subsection{Voice Circuit}

The system uses the ISD1420 speech chip of the company ISD of America. ISD1420 is a high qualified single voice record-replay circuit, which is made up with oscillator, voice store unit, pre-amplifier, AGC (automatic gain control) circuit, anti-interference filter, output amplifier. Voice circuit should finish prerecording, storing information, and playing voice while put through.

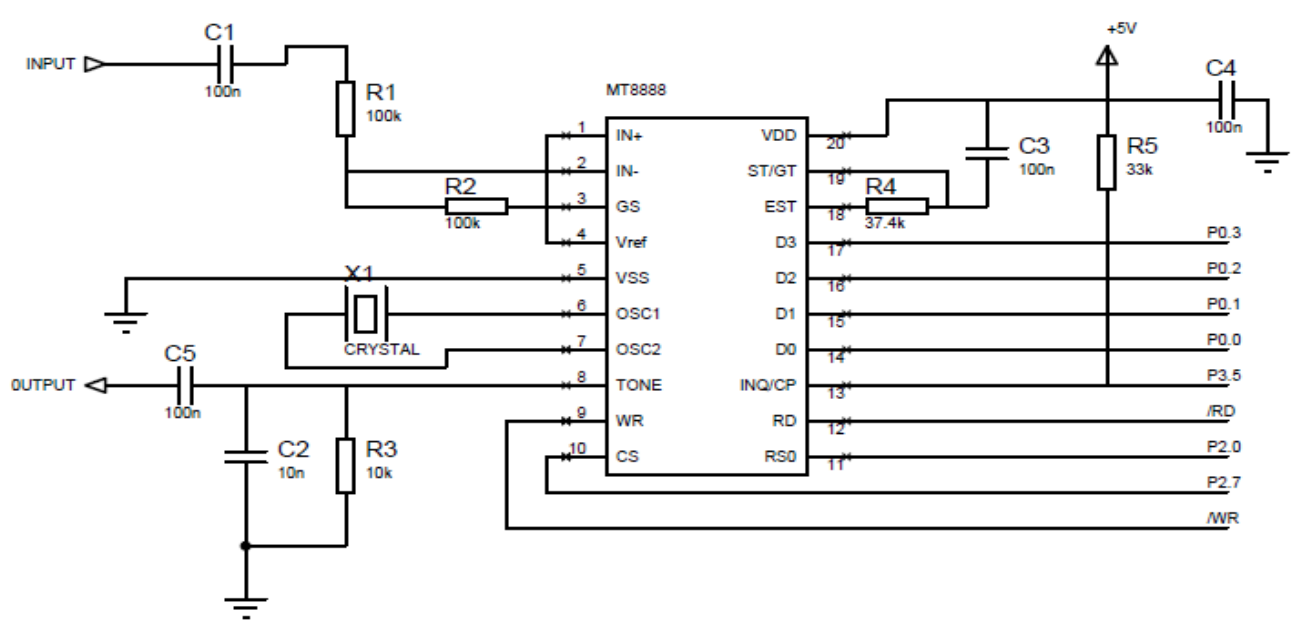

Figure 3.1. Mt8888 Interface Circuit 


\subsection{Telephone Interface Circuit}

Schematic diagram of phone interface circuit in the system is shown in figure3.2.

To ensure the normal sending of DTMF signal of dial circuit and normal broadcasting of voice signal of voice circuit, polar protection circuit constituted by diode bridge circuit, must be added in interface circuit.

According to the standard of mobile access by ministry of posts and telecommunications, direct current resistance should be less than or equal to $300 \mathrm{R}$ in off-hook, so stimulating off-hook and on-hook operation can be done by directly joining a high power resistance of 200R with polar protection circuit. Relay J2 actuation and Voice circuit work together to connect. In order to reduce systematic power dissipation, relay with high sensitivity and $+5 \mathrm{v}$ working voltage are chosen. Dial and voice circuit can connect with phone interface circuit via 1:1 isolation transformer to improve anti-interference of sound signal.

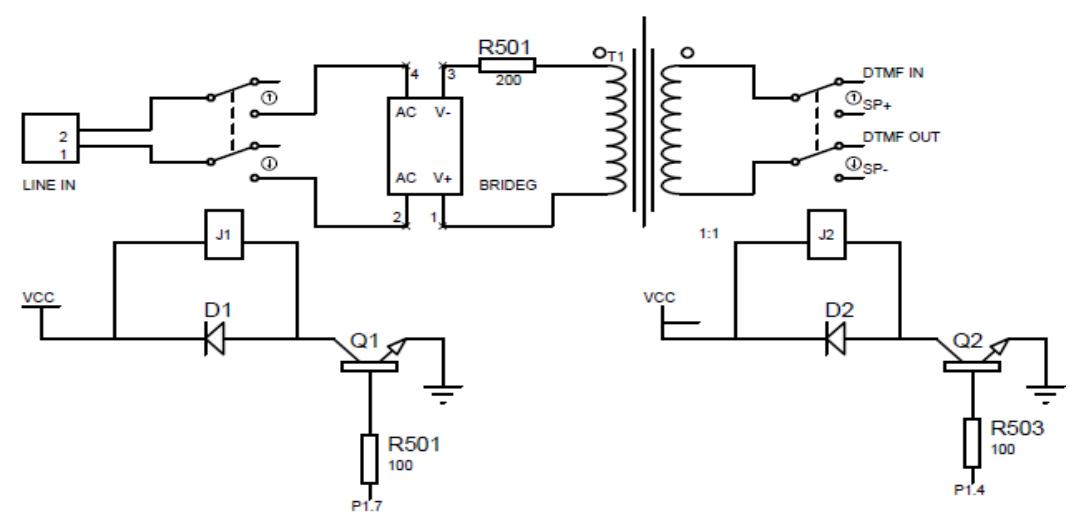

Figure 3.2. Telephone Interface Circuit

\subsection{Key Interface Circuit Design}

Keyboard as a main part of microcontroller application system is an obbligato input mode in man-machine interaction. It can finish inputting data and transferring command to microcontroller, inputting information by using the connection and break of mechanical contact. A key as a switch actually, using two 1/0 linear connection to judge the on-off state, this alertor has twelve keys, ten numeric keys, \# and *.we use 3X4 keyboard and seven 1/0 line. The system expands inlet/outlet $8255 \mathrm{~A}$ because of the limitation of $89 \mathrm{c} 51$ itself.3X4 links with the PC port of 8255A, C port PCO-PC3 as the line-scan output, C port PC4-PC6 as linear input, low level valid.

\section{Software Design}

Autoalarm adopts modular design, divided into master control modules, alarm and interrupt processing modules, dial, voice and keyboard input modules in the software part, MCS-51 assembly language in the programming.

\subsection{Main Program Design}

There is a $100 \mathrm{~ms}$ delay of microcontroller at first to make each pin restore after connecting power source. During initialization, the single chip opens external interruption 0 to restore each zone bit, and then scans that whether keyboard is pressed or not, operating relevant key disposal subroutine if pressed, continues to scan after disposition, and waits for the interrupt made by dangerous cases. Once such cases happening, interrupt service subroutine will perform its duty. 


\subsection{Interrupt Service Subroutine Design}

INTO foot of microcontroller is linked to burglarproof infrared sensor. In running period, main program initializes at first by opening the external interruption 0,then produces interruption and shifts to interrupt processing subroutine if dangerous cases happening. Two situations may lead to false alarm, first, infrared detection is easy to suffer external disturbance; second, our activities indoors may cause it for ignorance of disarming. In order to prevent such mistake, interrupt processing subroutine makes a delay to judge stealing and remove external disturbance for infrared detection and false alarm made by host's activity. If no stealing, then returning; if having stealing and missing disarming key, operating alter processing subroutine.

\subsection{Alarm Processing Subroutine Design}

Alter processing subroutine controls off-hook, tone judgment, dialing, voice weft sending, on-hook. Firstly, subroutine for off-hook is called to simulate off-hook and delaying to stabilize system. Then, subroutine of detection and judgment is called to distinguish dial tone. When there is no dial tone, subroutine on-hook will be called to call subroutine off-hook again after delaying for some time. When there is a dial tone, subroutine dialing will be called to dial the phone of host and then to analyze the tone on the caller side by invoke subroutine of detection and judgment. As for subroutine dialing: when the tone of caller side is busy tone, subroutine on-hook will be called and after a timer delay subroutine off-hook will be called. When ring-back tone can be heard on the caller side, subroutine of dialing will judge whether or not the callee is off-hook by calling subroutine of detection and judgment. If ring-back tone last more than 48 seconds, subroutine on-hook will be called, and then subroutine off-hook will be called after a time delay. If ring-back tone terminated in 48 seconds, a prerecorded alarm voice will be replayed by routine of calling voice, and at the same time subroutine of on-hook will be called. At last the next number will be dialed. This way all the numbers prestored will be dialed. All steps above will be repeated 3 times, before the program returns.

\subsection{Signal Detection And Judgment Modular Programming}

In order to detect tone, MT8880 need to be set as call processing mode, namely CP mode. Using this mode can MT8880 detect all tones, such as dial tone, busy tone, and ring-back tone. All tones belonging to $450 \mathrm{~Hz}$ center frequency and $250 \mathrm{~Hz}$ bandwidth can receive square wave signal representing tone from Schmitt trigger through filter selection and high-gain comparator amplitude limiting, and output in IRQ/CP port. Microprocessor analyzes calling process in IRQ/CP port. According to the standard of SPC (stored program control) exchange, dial tone adopts $450 \mathrm{~Hz}$ continuous sine wave, its positive-negative error is $25 \mathrm{~Hz}$; busy tone and ring-back tone all use $450 \mathrm{~Hz}$ sine wave, lasting $0.35 \mathrm{~s}$ and $1 \mathrm{~s}$ in on and off status. So we can judge the tone by counting exporting square wave of IRQ/CP foot according to this schema.

\subsection{Voice Modular Programming}

ISD512, a high qualified monolithic voice record-replay circuit produced by America ISD company, with the help of direct emulation storage technology (patent of ISD company) to realize recordings' deposition into permanent storage unit, offers zero power information storage and finish the reappearance of original voice. Microcontroller P2.3 controls playback, P2.3 changes from high level to low level, begins playback when it touches the playback foot of ISD1512, then it changes to high level to finish playback after 5 seconds. After that, system changes to the next status for another two times, and then eliminates alert zone bit. After all the above steps is done, system returns. 


\subsection{Dial Modular Programming}

Firstly initializing MT8880 and setting it as BURST schema; then dialing one after another until done. Setting MT8880 as DTMF schema when using autodial and working in unexpected way.

Initializer is as follows:

$\begin{array}{lr}\text { INT-MT8880: MOV } & \text { DPTR, \#7F00H } \\ \text { MOVX A, @DPTR } & \text {; Read Status Register } \\ \text { MOV A, \#00H } & \\ \text { MOVX @DPTR, A } & \text {; Write a control register } \\ \text { MOVX @DPTR, A } & \\ \text { MOV A, \#08H } & \\ \text { MOVX @DPTR, A } & \\ \text { MOV A,\#00H } & \\ \text { MOVX @DPTR,A } & \\ \text { MOVX A.@DPTR } & \\ \text { RET }\end{array}$

\subsection{Keyboard Modular Programming}

Keyboard modular program mainly finishes the verification and disposition of keys.

Keyboard, by which can finish inputting data and transferring command, is a critical component in microcontroller application system and an obbligato input mode in man-machine conversation. This alarm keyboard has twelve keys, ten numeric keys and two function keys $(*, \#), *$ is for setting monitor command and withdrawing monitor command (negation EXO), \# is for changing phone numbers stored in AT24C01.Keyboard formed to four rows and three ranks, first rank, from top to bottom are 1,2,3,4; second rank, 5,6,7,8; third rank, 9,0,\#, .scanning * at first, if * is not pressed, continuing to scan \#;if pressed, executing organizing or withdrawing a monitor, then scanning \# when finished. If \# is not pressed, system returns directly; otherwise subroutine GH will be called before return. The function of subroutine $\mathrm{GH}$ is to change phone numbers in AT24C01.

\section{Experiment}

\subsection{Experiment Design}

Conduct an imitate experiment on hardware and software unit in laboratory, if normal, then coming the real test on the whole alarm system at home. We can choose four different places to install probes orderly (Pyroelectric infrared probe) to conduct the experiment at home. The entrance was used as the first place (recorded as A), the near window as the second place (recorded as B), the drawing room as the third place (recorded as C), and the bedroom as the last place (recorded as D). After probes (2 meter tall) were installed, 3 different distances was chosen. Three different locations with the same distance were chosen as the detected people's location to experiment. We chose three different persons as the objects in the same detected place to have the experiment.

\subsection{Experimental Result And Analysis}

The specific experiment result is showed in table1. 
Table 1. Experimental Result Table

\begin{tabular}{|c|c|c|c|c|c|c|c|c|c|c|c|c|}
\hline \multirow{2}{*}{  } & \multicolumn{3}{|c|}{$2 \mathrm{~m}$} & \multicolumn{3}{|c|}{$4 \mathrm{~m}$} & \multicolumn{3}{|c|}{$6 \mathrm{~m}$} & \multicolumn{3}{|c|}{$8 \mathrm{~m}$} \\
\hline & $\begin{array}{l}\vec{\Xi} \\
\stackrel{8}{8}\end{array}$ & 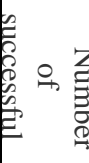 & 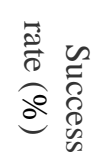 & 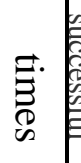 & $\begin{array}{ll} & \\
0 \\
0\end{array}$ & 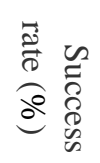 &  & 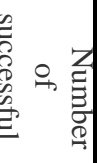 & 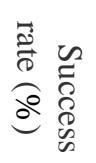 & 志. & 。 & 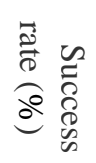 \\
\hline A & 9 & 9 & $\begin{array}{l}10 \\
0\end{array}$ & & 9 & $\begin{array}{l}10 \\
0\end{array}$ & & 9 & $\begin{array}{l}10 \\
0\end{array}$ & & 9 & $\begin{array}{l}10 \\
0\end{array}$ \\
\hline B & 9 & 9 & $\begin{array}{l}10 \\
0\end{array}$ & & 9 & $\begin{array}{l}10 \\
0\end{array}$ & & 9 & $\begin{array}{l}10 \\
0\end{array}$ & & 9 & $\begin{array}{l}10 \\
0\end{array}$ \\
\hline $\mathrm{C}$ & 9 & 9 & $\begin{array}{l}10 \\
0\end{array}$ & & 9 & $\begin{array}{l}10 \\
0\end{array}$ & & 9 & $\begin{array}{l}10 \\
0\end{array}$ & & 8 & 89 \\
\hline $\mathrm{D}$ & 9 & 9 & $\begin{array}{l}10 \\
0\end{array}$ & & 9 & $\begin{array}{l}10 \\
0\end{array}$ & & 9 & $\begin{array}{l}10 \\
0\end{array}$ & & 9 & $\begin{array}{l}10 \\
0\end{array}$ \\
\hline
\end{tabular}

Because choose 3 locations (three different locations with the same distance to the probe) as the detected people's location to experiment, we choose three different persons as the objects in the same detected place to have the experiment ;9 times experiment with the same distance .In table1, there one error. The reason for the error is Person's direction is radial movement, Infrared pyroelectric sensor for the radial movement the least sensitive, for the transverse direction (i.e., a direction perpendicular to the radius) movement is the most sensitive. Choosing the best detector installation location is very important to improve the sensitivity. According experiment specific result and analysis, we can conclude that the alarm system can detect people's normal actions in effective area.

\section{Conclusion}

The phone alarm based on microcontroller has strong practical value and broad market development prospects with features of simple structure, strong stability and low cost.

Innovation points of the author: storage and processing capacity of microcontroller enables the phone alarm to have higher technological content, shorter development cycle and lower cost.

\section{Acknowledgements}

This work was supported by the Key Science and Technology Program of He'nan Province of China under grant No 132102210044. It was also supported by Foundation of He'nan Educational Committee under grant No 14A520010.

\section{References}

[1] Y. Sun, "Design of intelligent remote controlling calendar", 19(SUPPL.2), (2011), pp. 298-304.

[2] X. Wang and Y. Zhao, "Design of real-time monitoring system on loading-intensity in fitness training", vol. 30, no. 7, (2010.), pp. 844-848.

[3] J. Fonollosa, et. al., "Ethylene optical spectrometer for apple ripening monitoring in controlled atmosphere store-houses", vol. 136, no. 2, (2009), pp. 546-554.

[4] J. A. Gazquez, et. al., "Intelligent telemetry watches cave visitors", vol. 1, no. 3, (2003), pp. 24-27.

[5] J. O. Peralta and M. T. C. Peralta, "Security PIDS with physical sensors", real-time pattern recognition, and continuous patrol, vol. 32, no. 4, (2002), pp. 340-346.

[6] J. Y. Wu, W. J. Li, J. P. Huang, J. L. Zhang and D. R. Chen, "Key techniques for Mobile Internet, a survey”, SCIENTIA SINICA Informationis, vol. 45, no. 1, (2015), pp. 45-69.

[7] J. Zhang, J.-Y Wu, "Provable Secure Efficient Arbitrated Quantum Signature Scheme", Journal of Beijing University of Posts and Telecommunications, vol. 36, no. 2, (2013), pp. 113-116. 


\section{Authors}

Guo Hong-Tao, received his MS degree in computer science from HUST (Hua-zhong University of Science and Technology), Wuhan, China in 2005. He is currently a lecturer in the software institute at North China University of Water Resources and Electric Power. He has more than 10 technical publications as well as about 3 patents. His current research interests include wireless communication system, 